Nucl. Med. Biol Vol. 18, No. 5, pp. 565-567, 1991

Int. J. Radiat. Appl. Instrum. Part B

(C) Pergamon Pres\$ plc 1991. Printed in Great Britain

$0883-2897 / 91 \$ 3.00+0.00$

\section{Improved Monoclonal Antibody Tumor/Background Ratios with Exchange Transfusions}

\author{
CHRISTINE A. HENRY', ANAIRA C. CLAVO' and \\ RICHARD L. WAHL ${ }^{1,2 *}$
}

University of Michigan Medical Center, Division of Nuclear Medicine, Departments of 'Internal Medicine and ${ }^{2}$ Radiology 1500 E. Medical Center Drive, Ann Arbor, MI 48109-0028, U.S.A.

\section{(Received 1 August 1990)}

Blood exchange transfusions were performed in nude rats with subcutaneous HTB 77 human ovarian carcinoma xenografts in an attempt to improve specific monoclonal antibody (MoAb) tumor/non-tumor uptake ratios. Animals were injected intravenously with both ${ }^{131} \mathrm{I}-5 \mathrm{G} 6.4$ specific and ${ }^{125}$ I-UPC-10 non-specific MoAb. Twenty-four hours later $65-80 \%$ of the original blood was exchanged with normal heparinized rat blood and then these rodents were sacrificed. Exchange transfusion significantly $(P<0.05)$ decreased normal tissue activities of ${ }^{131} \mathbf{I}$ (except for muscle) by $63-85 \%$, while tumor activity decreased only $5 \%$. Tumor to background ratios increased from $0.1-0.8$ to 2.3-6.3. Exchange transfusions substantially enhance tumor/normal tissue antibody uptake ratios and, along with plasmapheresis, may be useful in enhancing antibody localization in vivo, particularly for therapy.

\section{Introduction}

Radiolabeled monoclonal antibodies (MoAbs) are gaining utility in the diagnosis and treatment of some malignant tumors. Diagnostic imaging and therapeutic irradiation of tumors are most effective when using the MoAb that binds specifically to the target tissue without significant non-specific binding to normal tissues or excessive blood pool radioactivity. Although MoAbs have specific target affinities, such as the murine 1gG2ak 5G6.4 for ovarian carcinoma, they also exhibit significant non-specific binding in vivo which creates a substantial amount of background activity, making imaging and therapy difficult. Several approaches have recently been reviewed which aim to preserve tumor but reduce background activity, but each of these methods is in some sense a compromise and the localization of radiolabeled MoAbs to tumors remains less than ideal (Goodwin, 1987). For example, when antibody fragments are injected, the fragment clears from the blood more rapidly than intact antibody, thus allowing earlier imaging times and better tumor/background ratios, but the absolute radioantibody

*All correspondence should be addressed to: Richard L. Wahl, University of Michigan Medical Center, Nuclear Medicine, 1500 E. Medical Center Drive, Ann Arbor, MI 48109-0028, U.S.A. uptake into the tumor is lower than with intact antibody (Wahl et al., 1983).

In an alternate approach, Wahl et al. (1988a) were able to improve tumor/background ratios and enhance gamma camera imaging using systemic vascular perfusion with saline. Rats injected with $30 \mu \mathrm{Ci}{ }^{131}$ I-5G6.4 showed a $50-70 \%$ drop in normal organ activities following systemic perfusion with saline while nude mice treated similarly showed a 2.33-fold larger drop in background antibody uptake than in the tumor. This approach was conceptually related to that described by Begent et al. (1982), in which liposomally-entrapped second antibody directed against the first antitumor antibody was given. The resulting immune complexes then traffic to the spleen and liver for clearance. With this approach, increased splenic uptake can occur with radioiodinated antibodies and effective clearance of such radiometal labeled antibodies is likely not possible. Similarly, the use of anti-mouse antibodies given after the radiolabeled antibody can improve tumor/blood ratios, but result in increased targeting of immune complexes to the liver and spleen (Goldenberg et al., 1987; Wahl et al., 1987). The saline perfusion technique described by Wahl was able to improve tumor/non-tumor uptake ratios but it was difficult to perform in the mouse and was incompatible with life. The current study is an attempt to lower all background radioactivity levels without reducing the uptake in the tumor, using a more refined approach. Instead of incomplete perfusion with saline in a nude mouse, multiple blood exchange transfusion were performed on nude rats with subcutaneous HTB77 ovarian carcinomas, a procedure compatible with life and similar to plasmapheresis. The exchange transfusion technique is simpler to perform in rodents, however, and thus was evaluated. This physical removal of radioactive blood should remove a significant percentage of the non-specifically bound antibody from normal tissues, without decreasing the percent bound to the tumor, thus improving tumor/background ratios. These experiments test the feasibility of this concept.

\section{Methods}

\section{Monoclonal antibodies}

5G6.4 is an IgG2ak murine MoAb which localizes specifically to ovarian cancer xenografts when administered i.v. (Wahl et al., 1985, 1986b). It was radiolabeled by the iodogen method using $1.6 \mu \mathrm{g} \mathrm{Ab}: 1 \mu \mathrm{g}$ iodogen (Wahl et al., 1988a). The specific activity of the radioantibody injected was approx. $17 \mu \mathrm{Ci} / \mu \mathrm{g}$. The immunoreactivity of the antibody was tested in a direct $1 \mathrm{~h}$ cell binding assay (Wahl et al., 1990).

$\mathrm{UPC}-10$ is an IgG2ak murine MoAb but is not reactive with ovarian carcinoma and was purchased from Bionetics (Wahl et al., 1988b, 1989, 1990). It was labeled by the iodogen method using approximately the same antibody to iodogen ratio as the $5 \mathrm{G6.4} \mathrm{MoAb}$ (Wahl et al., 1987). Its specific activity was $8.3 \mu \mathrm{Ci} / \mu \mathrm{g}$. Free iodine was removed from protein bound iodine by anion exchange chromatography for both reagents. For both antibodies, free iodine levels in the preparation were assayed using silica gel thin layer chromatography.

\section{Animal studies}

Female nude rats (N:NIH-rnu) from Taconic Farms were innoculated with a single cell suspension of 10 million HTB77 (human ovarian carcinoma cell line from ATCC) cells subcutaneously in the skin over the left shoulder. Four to six weeks after injection the rats developed tumors of approx. $2 \mathrm{~cm}$ in diameter. Before injection with the $\mathrm{MoAb}$ 
mixture the right common carotid artery of experimental animals was cannulated with the end of a 7 in. long piece of Silastic tubing from Dow Corning $(0.020$ i.d. $\times 0.037$ in. o.d.). The control group was not chronically cannulated. This heparinized saline-filled tube was exteriorized through the back of the neck and housed in a small stainless steel button sewn to the skin to prevent damage by the animal. The chronic cannulation technique allowed for easier and more replicable vascular access during the transfusions and less radiation exposure for the researcher.

Both control and experimental animais were injected i.v. into the femoral vein with approx. $80 \mu \mathrm{Ci}$ each of ${ }^{131} \mathrm{I}-5 \mathrm{G} 6.4$ and ${ }^{125}$ I-UPC-10 (when possible) in a volume between 0.1 and $0.5 \mathrm{~mL}$. The procedural intensity of the exchange transfusions was such that only a limited number of animals could be studied per day. Thus, the experiments were conducted over several weeks. The animals were housed in individual cages and fed standard Purina Laboratory Rat Chow and tap water ad libitum.

Twenty-four hours after antibody injection the blood exchange was performed on the experimental group. The rats in both groups were anesthetized using $50 \mathrm{mg} / \mathrm{kg}$ Pentobarbital. Each exchange involved $2 \mathrm{~mL}$ of blood being removed from the nude rat and replaced with $2 \mathrm{~mL}$ of heparinized blood collected from normal Sprague-Dawley rats. A total of eight transfusions were performed with at least $10 \mathrm{~min}$ between transfusions, for a total replacement of between 65 and $80 \%$ of the original blood. This assumes total rat blood volume equals $50.0-64.1 \mathrm{~mL} / \mathrm{kg}$ (Pentel et al., 1987; Wang, 1959). Gamma camera imaging was performed when possible using a GE400 AT $\gamma$ camera with a high energy collimator interfaced to a GE STAR computer before and after perfusion. After the final transfusion, all the animals were sacrificed and tissue distribution studies performed by removing and weighing tissue samples and counting them at dual $\left({ }^{131} \mathrm{I}\right.$ and $\left.{ }^{25} \mathrm{I}\right)$ window settings in a Packard gamma counter (Wahl et al., 1984). Statistical analysis was by unpaired one tail $t$-test.

\section{Results}

After technical proficiency was established, the animals survived the multiple exchange transfusions well, without extra monitoring. The initial control and experimental animals received only ${ }^{131} \mathrm{I}-5 \mathrm{G} 6.4{ }^{13 !} \mathrm{I}-5 \mathrm{G} 6.4$ and ${ }^{125} \mathrm{I}-\mathrm{UPC}-10$ were given to the remainder of the animals.

The perfusion was reasonably complete, and using $\gamma$ camera imaging, whole body drops in ${ }^{131}$ I radioactivity of $40-50 \%$ were typical. Radioactivity levels per gram of tissue (\% $\mathrm{kg}$ injected dose $/ \mathrm{g}$ ) at the time of sacrifice are shown in Table 1 for ${ }^{131} I$ and in Table 2 for ${ }^{125} I$ for both control and experimental groups. These show that blood levels decreased approx. $80 \%$ with perfusion, however.

Comparison of the tissue biodistribution of activity $\left(a^{131} \mathrm{I}\right)$ in the control versus the exchange transfused groups shows significantly lower values followed perfusion for all tissues

Table 1. Biodistribution of ${ }^{131} \mathrm{I}-5 \mathrm{SG6.4}$ in exchange transfusion vs control rats

\begin{tabular}{lccc}
\hline Tissue & $\begin{array}{c}\text { Controls }(n=7) \\
(\% \mathrm{~kg} \text { inj. dose } / \mathrm{g})\end{array}$ & $\begin{array}{c}24 \mathrm{~h} \text { Exchange }(n=5) \\
(\% \mathrm{~kg} \text { inj. dose/g) }\end{array}$ & $\begin{array}{c}\% \text { Drop in } \\
\text { activity }\left(^{131} \mathrm{I}\right)\end{array}$ \\
\hline Blood & $0.931 \pm 0.201$ & $0.195 \pm 0.073^{*}$ & 79.0 \\
S. bowel & $0.161 \pm 0.031$ & $0.066 \pm 0.028^{*}$ & 59.0 \\
Ovary & $0.271 \pm 0.049$ & $0.094 \pm 0.032^{*}$ & 65.3 \\
Liver & $0.327 \pm 0.103$ & $0.049 \pm 0.015^{*}$ & 85.0 \\
Kidney & $0.241 \pm 0.047$ & $0.056 \pm 0.019^{*}$ & 76.8 \\
Lung & $0.503 \pm 0.103$ & $0.141 \pm 0.061^{*}$ & 72.0 \\
Spleen & $0.163 \pm 0.022$ & $0.054 \pm 0.022^{*}$ & 66.9 \\
Heart & $0.180 \pm 0.032$ & $0.067 \pm 0.024^{*}$ & 62.8 \\
Muscle & $0.143 \pm 0.056$ & $0.093 \pm 0.073$ & 35.0 \\
Tumor & $0.135 \pm 0.019$ & $0.128 \pm 0.019$ & 5.2 \\
\hline
\end{tabular}

* Significantly $(P<0.05)$ less than control values.
Table 2. Biodistribution of ${ }^{125} \mathrm{I}-\mathrm{UPC}-10$ in exchange transfusion vs control rats

\begin{tabular}{lccc}
\hline Tissue & $\begin{array}{c}\text { Controls }(n=6) \\
(\% \mathrm{~kg} \text { inj. dose } / \mathrm{g})\end{array}$ & $\begin{array}{c}24 \mathrm{~h} \text { Exchange }(n=4) \\
(\% \mathrm{~kg} \mathrm{inj.} \mathrm{dose} / \mathrm{g})\end{array}$ & $\begin{array}{c}\% \text { Drop in } \\
\text { activity }\left({ }^{131} \mathrm{I}\right)\end{array}$ \\
\hline Blood & $0.958 \pm 0.275$ & $0.181 \pm 0.108^{*}$ & 81.1 \\
S. bowel & $0.161 \pm 0.041$ & $0.066 \pm 0.035$ & 59.0 \\
Ovary & $0.271 \pm 0.068$ & $0.094 \pm 0.041^{*}$ & 65.3 \\
Liver & $0.216 \pm 0.046$ & $0.049 \pm 0.015^{*}$ & 77.3 \\
Kidney & $0.229 \pm 0.054$ & $0.056 \pm 0.015^{*}$ & 75.5 \\
Lung & $0.522 \pm 0.126$ & $0.141 \pm 0.067^{*}$ & 73.0 \\
Spleen & $0.185 \pm 0.027$ & $0.054 \pm 0.051$ & 70.8 \\
Heart & $0.189 \pm 0.043$ & $0.067 \pm 0.032^{*}$ & 64.6 \\
Muscle & $0.169 \pm 0.063$ & $0.093 \pm 0.095$ & 45.0 \\
Tumor & $0.108 \pm 0.017$ & $0.082 \pm 0.024$ & 24.1 \\
\hline
\end{tabular}

*Significantly $(P<0.05)$ less than control values.

except muscle and tumor $(P<0.05)$. The absolute amount of activity in the tumor dropped little, $5 \%$, compared with the drop in the normal tissues of $63-85 \%$ (Table 1), thus improving tumor/background ratios from $0.1-0.8$ to $2.5-6.3$.

The tissue distribution of ${ }^{125} I$ activity shows significantly lower values in the exchange transfused group for the blood, ovary, liver, kidney, lung and heart. The small sample size may be one reason for the limited difference between the exchange transfused group and controls for this antibody ( ${ }^{125}$ I-UPC-10). Tumor/blood ratios in the experimental group were $1.39 \pm 0.53\left({ }^{131} \mathrm{I}-5 \mathrm{G} 6.4, n=3\right)$ and $0.57 \pm 0.16$ ( ${ }^{125}$ I-UPC-10, $n=3$ ). In the control group the ratios were $0.22 \pm 0.07$ ( $\left.{ }^{131} \mathrm{I}-5 \mathrm{G} 6.4, \quad n=7\right)$ and $0.24 \pm 0.13$ $\left({ }^{125} \mathrm{I}-\mathrm{UPC}-10, n=5\right.$ ).

\section{Discussion}

Blood exchange transfusions $24 \mathrm{~h}$ post-MoAb injection substantially improve tumor/background ratios of specific radiolabeled MoAb delivered i.v, to rodents with subcutaneous human ovarian carcinomas. Exchange lowered nearly all normal tissue activity without a substantial change in the binding to tumor. The drop in blood activity ${ }^{131} \mathrm{I}$, 5G6.4) was greater than $75 \%$ and in other tissues (except muscle) it was between 63 and $85 \%$ while the tumor dropped only $5 \%$. This clearly shows that exchange transfusion can greatly reduce background activity thereby improving tumor/background ratios. Such enhancements would undoubtedly improve tumor imaging as well as enhance specific delivery of the MoAb to its target. This lesser decline in normal muscle was also seen when saline perfusion was performed and may be due to the relatively low vascular volume in muscle.

Most likely improved tumor/background ratios are possible because of specific binding by the MoAb to target sites on tumor cells as opposed to non-specific random or low affinity associations. Such non-specific interactions are more easily disturbed by perfusion with unlabeled blood in the normal tissues, whose activity levels dropped after exchange transfusion on average of $67 \%$ as compared to the tumor activity drop of $5 \%$. It is also possible that a component of this enhancement effect is duc to poor tumor vascularity (i.e. it is difficult to get radioantibodies into or out of tumors rapidly). It would seem that vascular permeability is only a partial contribution, however, because of the more rapid clearance of the non-specific antibody from the tumor ( $24 \%$ drop) as well as from the normal tissues $(68 \%$ drop). Additional studies in larger animals or man could further refine the specifics of the approach, such as the ideal time for blood exchange transfusion and the duration of the benefit, and the number of times the procedure could be performed.

This study demonstrates that it is possible, using blood exchange, to remove a substantial quantity of radioactivity from the blood and other normal tissues and improve 
tumor/background ratios. This improvement has been achieved without increasing levels of radioactivity in normal tissues such as the liver or spleen as can occur when polyclonal antimouse antibodies are used to clear the radioactivity (Goldenberg et al., 1987). The remaining questions include determining the feasibility of plasmapheresis techniques (Euler et al., 1985; Charlton et al., 1983) which are more applicable to clinical use than blood transfusions and whether radionuclide images and therapies improve sufficiently as a result of such improved tumor/background ratios to justify such an aggressive approach. Nonetheless, this exchange transfusion approach, or mimicry of plasmapheresis, may be of value in the treatment of cancer with radioantibodies and lays a framework for subsequent studies.

Acknowledgements--This work is supported in part by Grants CA33802 and CA41531. The excellent secretarial assistance of Ms Cathy Ruel is noted.

\section{References}

Begent R. H. J., Keep P. A., Green A. J. et al. (1982) Liposomally entrapped second antibody improves tumor imaging with radiolabeled (first) antitumor antibody. Lancet 2, (8301), 739-742.

Charlton B., Schindhelm K. and Farrell P. C. (1983) Effect of extracorporeal IgG removal on IgG kinetics. Trans. Am. Soc. Artif. Intern. Organs 29, 724-729.

Euler H. H., Krey U., Schröder O. and Löffler H. (1985) Membrane plasmapheresis technique in rats. Confirmation of antibody rebound. J. Immunol. Methods 84, 313-319.

Goldenberg D. M., Sharkey R. M. and Ford E. (1987) Anti-antibody enhancement of iodine-131 anti-CEA radioimmunodetection in experimental and clinical studies. J. Nucl. Med. 28, 1604-1610.

Goodwin D. A. (1987) Pharmacokinetics and antibodies. J. Nucl. Med. 28, 1358-1362.

Pentel P. R., Keyler D. E., Gilbertson D. G., Ruth G. and Pond S. M. (1987) Pharmacokinetics and toxicity of high doses of antibody Fab fragments in rats. Drug Metab. Dispos. 16, 141-145.

Wahl R. L. and Fisher S. (1987) Intraperitoneal delivery of monoclonal antibodies: enhanced regional delivery advantage using intravenous unlabeled anti-mouse antibody. Nucl. Med. Biol. 14, 611-615.

Wahl R. L. and Liebert M. (1989) Improved radiolabeled monoclonal antibody uptake by lavage of intraperitoneal carcinomatosis in mice. $J$. Nucl. Med. 30, 60-65.

Wahl R. L., Sherman P. and Fisher S. (1984) The effect of specimen processing on radiolabeled monoclonal antibody biodistribution. Eur. J. Nucl. Med. 9, 382-384.

Wahl R. L., Tuscan M. C. and Botti J. M. (1986a) Dynamic variable background subtraction: a simple means of displaying radiolabeled monoclonal antibody scintigraphy. J. Nucl. Med. 27, 545-548.

Wahl R. L., Liebert M., Biesman B., Roberts J., Jackson G., Kronberg S. and Laino L. (1986b) Production and characterization of a murine monoclonal antibody reactive with ovarian and other epithelial carcinomas. PAACR 27, 355.

Wahl R. L., Geatti O., Liebert M. and Laino L. (1985) Radioimmunoimaging and localization of human ovarian carcinoma xenographs. Radiology 157(P), 99.

Wahl R. L., Wissing J., delRosario R. and Zasadny K. (1990) Inhibition of autoradiolysis of radiolabeled monoclonal antibodies by cryopreservation. J. Nucl. Med. 31, 84-89.

Wahl R. L., Piko C. A., Beers B. A., Geatti O., Johnson J. and Sherman P. (1988a) Systemic perfusion: a method of enhancing relative tumor uptake of radiolabeled monoclonal antibodies. Nucl. Med. Biol. 15, 611-616.

Wahl R. L., Barrett J., Geatti O., Liebert M., Wilson B. S., Fisher S. and Wagner J. G. (1988b) The intraperitoneal delivery of radiolabeled monoclonal antibodies: studies on the regional delivery advantage. Cancer Immunol. Immunother. 26, 187-201.

Wang L. (1959) Plasma volume, cell volume, total blood volume, and $F$ cells factor in the normal and splenectomized sherman tat, Am. J. Phys. 196, 188-192. 